\title{
From Epilogue to Prologue: Chaos and the Arrow of Time
}

$\mathrm{I}$

LyA PRIgOgine and Isabelle Stengers's Order out of Chaos (1984) should carry a warning label: "CAUTION: Use at own risk. Authors have speculated beyond data. Conclusions are conjectural." The disclaimer is necessary because this book is not, as it may appear to be, simply a popularized version of the new sciences. Rather, it is an ambitious synthesis that goes well beyond what many scientists working in chaos theory would be willing to grant are legitimate inferences from their work. As speculative theory, it attempts to define the deeper significance-one might almost say the metaphysics - of an emerging area of research. At issue is whether chaos should be associated with the breakdown of systems or with their birth. Viewed as the epilogue to life, chaos almost inevitably has negative connotations. Seen as prologue, it takes on more positive evaluations.

At the center of Prigogine and Stengers's project is their view of how the new science relates to time. According to them, chaos theory provides a resolution to the long-standing philosophical debate about whether being or becoming is the essential reality. They point out that classical physics imagines the universe as reversible, able to move either backward or forward in time. The biological and human sciences, by contrast, locate themselves within an irreversible world of birth and mortality. Prigogine and Stengers see thermo- 
dynamics as forming a natural bridge between these views. At equilibrium, thermodynamic equations are classical in the sense that they are reversible, but far from equilibrium they are irreversible and hence bound to a one-way direction in time. A thermodynamics that integrates reversibility with irreversibility would, they assert, allow being and becoming finally to be reconciled.

Even from this brief summary, the reader can appreciate the disparity between the sweep of the theoretical claims and the technical basis on which they rest. To imagine that profound philosophical questions can or should be answered by advances in irreversible thermodynamics is apt to evoke uneasiness among most scientists and outright skepticism among humanities scholars. Yet there have been scientific theories that have significantly affected social attitudes-evolution, relativity, and quantum mechanics, for example. Whether chaos theory will have this kind of impact remains to be seen. Even more problematic, in my view, is whether the new paradigms are causes of social change or are themselves reflections of larger cultural currents. In light of these uncertainties, it is especially important to be clear about what different research programs within the sciences of chaos have accomplished, and what they have so far merely promised or suggested.

The even tone in which Order Out of Chaos is written does not encourage this distinction. Facts that have been extensively verified are presented in the same manner as speculations backed by little or no experimental confirmation. Only once do Stengers and Prigogine acknowledge that some of their scientific claims are conjectural. At the end of the chapter on microscopic interpretations of irreversibility, they add a "word of caution" that "experiments are in preparation to test these views," and as "long as they have not been performed, a speculative element is unavoidable" (p. 290). The implication, of course, is that the "speculative element" waits only on the completion of experiments to be removed. Since no attempt is made in the chapter to identify why, or even what, points are speculative, the lay reader has no way to evaluate the claim that experimental confirmation is forthcoming. The "word of caution" is just that-a brief comment added as an afterthought, not an acknowledgment that many of the chapter's conclusions are controversial.

Additional difficulties are presented by an inference many readers have drawn from Order out of Chaos, that Prigogine is as central to 
chaos theory as Darwin was to evolution or Einstein to relativity. To someone who works in the field, it appears that Prigogine's work has supplied a few rooms in a large building. Certainly he did not establish the building's architecture, nor did he single-handedly lay the foundation. To be fair, the misapprehension is not entirely his fault. It is perhaps understandable that in a text he co-authored, his research would be foregrounded and other research programs reported as though they merely provided corroborating evidence for his thesis. Nevertheless, the imbalance implicit in Order out of Chaos should be pointed out and corrected, not replicated.

Despite these problems, Order out of Chaos is an important work. It is frequently cited by scholars interested in connections between literature and science because it is one of the very few texts available that attempt to explain the new sciences in words rather than equations. Moreover, the research it reports is interesting and worthwhile, independent of its philosophical significance. But as we shall see, its most important claims have to do with changes in world view rather than shifts in scientific theorizing. To see how these claims emerge, it will be necessary to review Prigogine's contributions to irreversible thermodynamics. For that we need to return to the second law of thermodynamics.

As we saw in chapter 2, the second law led to predictions of a "heat death" in which the universe, having exhausted all heat reservoirs, degenerates to a state of maximum dissipation. This scenario contrasts sharply with the spontaneous increase of organization in living organisms, as in the increasing differentiation of an embryo. Twenty years after Kelvin formulated the idea of "universal dissipation," he agonized in print over whether living organisms were an exception to it. He finally concluded it was futile to worry about the matter. "But the real phenomena of life infinitely transcend human science," he wrote, "and speculating regarding consequences of their imagined reversal is utterly unprofitable" (I9II: I2). The apparent discrepancy between entropic decay and biological complexity can be reconciled when one recognizes that the second law applies only to closed systems, whereas all living organisms are open systems that continually receive energy from outside themselves. Nevertheless, the contrast was troubling to Kelvin, and remained so for many thermodynamicists. Biologists, for their part, tended to feel that thermodynamics was largely irrelevant to their concerns. 
Prigogine gave a new twist to this old problem by reformulating the way entropy production takes place. He reasoned that the overall entropy term can be divided into two parts. The first reflects exchanges between the system and the outside world; the second describes how much entropy is produced inside the system itself. The second law requires that the sum of these two parts be positive, except at equilibrium, when it is zero. But if the system is very far from equilibrium, the first term will be so overwhelmingly positive that even if the second term is negative, the sum can still be positive. This means that, without violating the second law, systems far from equilibrium can experience a local entropy decrease. For systems of interest to Prigogine, this decrease manifests itself as a dramatic increase in internal organization. To foreground the connection between self-organizing processes and large entropy production, Prigogine called such reactions "dissipative" systems (Nicolis and Prigogine, 1977). He knew that for Kelvin and other thermodynamicists, this word connoted waste, decline, and death. By using it to denote the spontaneous appearance of organized structure, Prigogine emphasized the important positive role that entropy production can play.

The problem then was to understand how self-organization takes place. Extensive research was undertaken on such chemical processes as the Belousov-Zhabotinskii (BZ) reaction, which exhibits dramatic and highly organized structures far from equilibrium. The primary and secondary reactions involved in the BZ reaction are complex, but the reaction itself is not difficult to produce. All one needs is a petri dish that contains bromate and cerium ions in an acidic medium. The reaction is sufficiently dramatic to warrant describing it as if we were there. What would we see?

Initially the solution appears homogeneous. Suddenly a colored ring forms near the dish's center and begins to pulsate, spreading outward toward the perimeter.' We discover that if we jiggle the

'The ring is visible because the catalyst for the reaction is a colorimetric indicator. A distinctive feature of the $B Z$ reaction is that the catalyst for the reaction is produced by the reaction itself. When a reaction product feeds back into the reaction in this way, the reaction is said to be autocatalyzing. The feedback loops involved in auto- and cross-catalyzation are characteristic of self-organizing reactions. They provide the mechanisms necessary for the reactions to vacillate between intermediate stages, instead of proceeding immediately from reactants to products. 
dish, ruffle our beards over it (as chemists of old used to do), or drop in dust particles, other rings form and fight with the first ring for dominance. Usually the ring oscillating at the highest frequency wins, progressively destroying the waves of lower-frequency oscillators until it is the only one to remain. If we dip a pencil into the dish and "break" a ring, it transforms into a spiral that begins to spin. We find that these fascinating displays do not last very long. As secondary reactions drain the chemicals away from the primary reactions, the displays gradually fade. Setting up the reaction again, this time we stir the reaction. We discover that the continuously stirred reaction creates a display that can sustain itself indefinitely. The stirred solution is originally homogeneous, as the first reaction was. Soon, however, the solution flashes blue, then red, then blue again, at regular intervals. We are seeing a second version of the $\mathrm{BZ}$ reaction that produces a so-called chemical clock.

Self-organizing reactions have been known for some time; the BZ reaction was recognized in Russia about thirty years ago by the two chemists who gave their names to it, Belousov and Zhabotinskii. Prigogine did not invent these reactions. Nor did he do the pioneering work of cataloguing their remarkably various forms; the credit for this work goes to Arthur Winfree and others. ${ }^{2}$ What Prigogine did was create a metaphysics that places these reactions at the center of a new view of how order emerges. Prigogine demonstrated mathematically that when initial concentrations of the reactants are large, the solution becomes unstable, and local fluctuations appear in the concentration of reagents. ${ }^{3}$ Past some critical point, these microscopic fluctuations correlate with other fluctuations spatially removed from them and become the central points from which arise the macroscopic rings and spirals of the $\mathrm{BZ}$ reaction. Prigogine and Stengers represent this coordination as macroscopic concentrations "instructing" local regions to engage in a process of self-organiza-

'For a review of the BZ reaction, see Arthur T. Winfree, "Rotating Chemical Systems," Scientific American 230 (1974): 82-95. Arthur Goldbeter and S. Roy Caplan discuss self-organizing reactions in biology in "Oscillatory Enzymes," Annual Review of Biophysics and Bioengineering 5 (1 976): 449-73.

"An accessible treatment of the mathematics can be found in Jeffrey E. Froehlich, "Catastrophe Theory, Irreversible Thermodynamics, and Biology," Centennial Review 28 (1984): 228-25I, especially pp. 233-235. 
tion through some sort of "communication" between them (1984: 63-89).

Prigogine's explanation is distinct from previous explanations because it does not rely on specific reaction mechanisms, such as stereospecificity, to explain how self-organization takes place. If anything, Prigogine and Stengers overemphasize this aspect of Prigogine's work, implying that it is extraordinary, even miraculous, that millions of individual molecules can "communicate" in such a way as to make macroscopic structures possible. Because they do not distinguish clearly between communication unique to living beings and "communication" between molecules, the rhetoric of Order out of Chaos takes on an anthropomorphic quality quite different from what one finds in most articles on chaos.

With this general background, we are now ready to consider what Prigogine and Stengers call the "leitmotif" of their text, the integration of being and becoming. Even a casual reading reveals that the chapter on microscopic irreversibility is crucial to the book's argument. Throughout earlier chapters, important questions are deferred to it; afterward, frequent references are made back to it. The chapter is indeed central, for it undertakes to integrate the timeless world of classical physics with the time-bound world of biology and chemistry. At issue is whether time's one-way direction is intrinsic to reality or an artifact of the observer.

In tackling the question, Prigogine and Stengers attempt to strengthen the case for time's irreversibility by reinterpreting the second law. The second law is involved because it gives time its arrow by defining "forward" as the direction of increasing entropy. Suppose you see a movie in which scattered pool balls magically reassemble into the triangular shape of the rack. You know the film is being run backward, because in real life systems never work like this. Chocolate poured into milk never spontaneously sorts itself into syrup again; ink smudged on a page never flows back again into a single clean line. These results are consistent with the second law's decree that entropy always tends to increase in closed systems. It is important to understand that this tendency is not predicted by classical physics. In Newton's equations of motion, negative time can be substituted for positive time and the equations still hold true. As far as Newton was concerned, time could run backward. The same is 
true for the special theory of relativity; it too treats time as a twoway street. Only the second law puts a point on time's arrow.

For years scientists and philosophers had pondered whether time's forward direction was merely very probable or absolute. The question arose because it was not clear whether the second law was a generalization about what would probably happen or intrinsic to microscopic reality. If the second law was merely probable, then the forward motion of time had an unavoidable subjective element. To see why, consider the pool-ball example again. Once the balls have been scattered from their original triangular formation, for time to run backward they must collide in just the right way so as to form the triangle again. If they form any other pattern (or no pattern at all), from our point of view time would not be running backward. The probability that this one specific pattern will occur is infinitesimally small, because it is only one of millions of possibilities. Although nothing inherent in physical reality causes time to move in one direction, it goes forward and not backward because the probability for events to happen in a myriad of different ways is infinitely greater than for them to happen in one way. And why must things happen in just this way? Because our knowledge defines the triangular pattern, and only the triangular pattern, as "past." Hence the necessity for time to move forward is in this view inherently subjective, an artifact of the observer's presence.

Prigogine and Stengers try to refute this argument by locating irreversibility in microscopic reality rather than in the observer's knowledge. They assert that once the balls are dispersed, the information the triangular form represents has been dissipated. For the pool balls to reassemble, they would have to "communicate" with each other about position, momentum, trajectory, and so on, so that all the different motions would be coordinated in just the right way. Even for a few pool balls, the volume of information involved is very large. If the entire universe were to run backward, it would be essentially infinite. Thus Prigogine and Stengers conclude that time can go only forward because an infinite information barrier divides past from present. In this view the second law is as much a statement about lost information as it is about dissipated energy. Just as some energy is dissipated in every real heat exchange, so some information is lost in every real matter/energy exchange. 
To see the force of this argument, imagine that when the balls begin retracing their trajectories, your body experiences a similar reversal of all the processes that occurred during the time you watched the balls disperse. If time could pivot and begin running backward in this way, you would be growing younger while the balls were reassembling. For this to happen, each particle in your body which had been involved in a collision while the balls were dispersing would have to "communicate" with every other particle it hit, so that all of these collisions could now happen in reverse. This would require millions more bits of information than the pool balls needed for their reassembly. If not just you and the balls but everyone else in the world was experiencing a similar reversal, the information required would be unthinkably enormous. Even so, we would have accounted for just a few minutes in the lives of just one species on one smallish planet in one galaxy. For time to run backward throughout the universe, the information would be so staggeringly huge that it is easy to see why Prigogine and Stengers call it an infinite barrier. The problem with time going backward, then, is not just in the observer's knowledge. Rather, it is in the massive correlation of information necessary to have collisions on every level, from subatomic particles to cars on a California freeway to meteorites striking Jupiter, reverse themselves.

To make this argument, Prigogine and Stengers find it convenient to introduce the idea (developed by Ludwig Boltzmann in the late nineteenth century) of correlated and uncorrelated particles. Before two particles collide, they have no necessary correlation with each other. The collision might or might not occur, depending on everything happening around them. The BMW might or might not hit the Honda, depending on whether the Chevette manages to swing around the truck blocking the path ahead of them. But once the BMW has hit the Honda, these two cars must be correlated if a time reversal is to occur; and all the collisions that preceded that collision must also be correlated, on every level, back to the beginning of time. When time goes forward there is a role for chance, because small or random fluctuations near a bifurcation point can cause a system to take a different path than it otherwise would. Whether the Chevette misses the truck could depend on whether a butterfly fluttering nearby distracts the driver's attention for a second. But when 
time runs backward along the same track it took before, every juncture point is already predetermined, and hence chance can play no further part in the system's evolution.

The introduction of chance hints at what is at stake in this argument. Compared to the question whether death is inevitable, the debate over whether the second law is an artifact of the observer or rooted in microscopic irreversibility may seem trivial. Prigogine and Stengers's point, however, is that these concerns are very much related. Humankind has always sought ways to come to terms with its mortality, and one of the ways that figured importantly in the history of science was to envision reality as a timeless realm purged of the irreversible changes that mark human experience. Prigogine and Stengers imply that, on a deep level, this view of reality was motivated by a desire to escape from an existence that seemed all too vulnerable to the vagaries of chance.

Few have felt the appeal of a timeless realm more deeply than Einstein. In his famous mediation on the "Temple of Science," Einstein speculated that the scientist or artist is attracted to his vocation because he is in

flight from everyday life with its painful harshness and wretched dreariness, and from the fetters of one's own shifting desires. A person with a finer sensibility is driven to escape from personal existence and to the world of objective observing and understanding. This motive can be compared with the longing that irresistibly pulls the towndweller away from his noisy, cramped quarters and toward the silent, high mountains, where the eye ranges freely through the still, pure air and traces the calm contours that seem to be made for eternity.

With this negative motive there goes a positive one. Man seeks to form for himself, in whatever manner is suitable for him, a simplified and lucid image of the world, and so to overcome the world of experience by striving to replace it to some extent by this image. [Einstein, I.954: 224-227]

It may seem strange that these lines were written by the man who formulated relativity theory. However, Einstein was led to relativity precisely because he saw it as the only way to preserve the overall invariance of physical laws. Before he settled on "relativity," he had considered calling his discovery the "theory of invariance." The appeal that he felt in "the calm contours that seem to be made for eternity" can be sensed when one looks at a Minkowski diagram of 
spacetime. ${ }^{4}$ Because time is one of the coordinates presented for inspection, there is a tendency to feel one is looking at reality from an eternal viewpoint. The illusion speaks to the strong sense Einstein had of a scientific model as a "simplified and lucid image of the world," beautiful in its exactness and clarity.

From this perspective, the assertion that the second law is an artifact of subjective experience is crucial. For if, on the contrary, the second law were intrinsic to reality, then irreversible change would be inextricably woven into the fabric of the universe, and it would be impossible to maintain (or create) an anywhen free from the taint of mortality. The subtext for the debate over the second law is thus a struggle over how one is to see reality on a fundamental level: bombarded by chance, subject to decay, headed toward death; or existing in a timeless realm where chance and irreversibility are merely subjective illusions.

We can now understand the full scope of the intervention Prigogine and Stengers want to make in Chaos out of Order. They insist that since irreversible events are demonstrably part of the world, in fact a much larger part than reversible ones, we must recognize that the view of classical physics is at best incomplete. Moreover, they suggest that the price paid for this classical perspective is alienation, for a timeless realm is a world divorced from human experience. In its place they offer a vision that they find both truer to reality and less alienating for the human spirit. The essential change is to see chaos as that which makes order possible. Life arises not in spite but because of dissipative processes that are rich in entropy production. Chaos is the womb of life, not its tomb.

There is evidence to support this view. Estimates indicate that the time required for life to arise if it relied purely on random sequencing of amino acids would be far longer than the present age of the universe (Madore and Freedman, 1987: 253). The probability, then, is that some kind of self-organizing process arose from chance fluctuations. The self-organizing process was able to proceed much

${ }^{4}$ Hermann Minkowski, a mathematician, was the first to realize that Einstein's special theory of relativity implied a four-dimensional spacetime. In 1908 he predicted that "space by itself and time by itself are doomed to fade away into mere shadows, and only a kind of union of the two will preserve an independent reality" ("Space and Time," in The Principles of Relativity [New York: Dover, 1908]). 
more quickly than random sequencing because it possessed the ability to create and replicate form. From this perspective, one could say that the second law in a sense created life, for only a world rich in dissipative processes would have been able to support self-organization. To make the second law intrinsic to reality is in this view comforting rather than threatening, for it suggests that the evolution of life was not an accident but a response to the essential structure of the cosmos.

This is why Prigogine and Stengers do not want to concede objectivity to those who, like Einstein, privilege a universe where irreversible change is an illusion. Instead, they imagine a world that is both "objective and participatory" (p. 299). They imply that we can know the world because it is constituted through the same processes that created and continue to govern human life. The passage they quote from Merleau-Ponty about a "truth within situations" aptly summarizes their position:

As long as I keep before me the ideal of an absolute observer, of knowledge in the absence of any viewpoint, I can only see my situation as being a source of error. But once I have acknowledged that through it I am geared to all actions and all knowledge that are meaningful to me, and that it is gradually filled with everything that may $b e$ for me, then my contract with the social in the finitude of my situation is revealed to me as the starting point of all truth, including that of science and, since we have some idea of the truth, since we are inside truth and cannot get outside it, all that I can do is define a truth within the situation. [Prigogine and Stengers, . 1984: 299]

Merleau-Ponty of course speaks from the perspective of a psychologist and phenomenologist, so it is perhaps not surprising that he would think in terms of a "contract with the social." It is a further stretch for Prigogine and Stengers to associate chemical processes with social reality. Yet their rhetoric is designed to make such a passage easy or plausible. On a deep level, this is no doubt why they tend to write about chemical reactions in anthropomorphic terms. They would make common cause with theorists within the human sciences who challenge totalizing views and celebrate the aleatory and stochastic. Although they approach the subject with a light touch, Prigogine and Stengers clearly agree with Michel Serres that implicit in traditional scientific objectivity is a will toward mastery 
which feeds on the illusion that the world is separate from us. ${ }^{5}$ Only if we blinded ourselves to the complex feedback loops characteristic of self-organizing structures could we think that we could exploit and dominate others without ourselves being profoundly affected by these actions.

These are heady conclusions. And if they are based solely on the scientific data Prigogine and Stengers present, they are shaky ones. Not only does their argument go far beyond the scientific data; it does not sit altogether easily with the data. Recall that the centerpiece of their interpretation of the second law is the infinite information barrier separating past from future. Yet their analysis of selforganizing structures emphasizes the seemingly miraculous way in which millions of molecules can "communicate" with one another. If molecules can engage in "communication" to build self-organizing structures, why can they not "communicate" to cause time to go backward? One could respond, of course, that self-organizing structures have been observed in nature, and that time reversal has not (at least not unequivocally). But this is to place the case on an empirical rather than a theoretical basis, which is precisely the aspect of traditional interpretations of the second law they were trying to overcome.

A further difficulty arises from the way Prigogine and Stengers group together research programs that are quite different in their assumptions, methodologies, and conclusions, as though all of this research points unambiguously toward an order-out-of-chaos paradigm. For example, they mention Feigenbaum's one-dimensional mappings (discussed in chapter 6) without bothering to explain the nontrivial differences that exist between mappings and multidimensional phase diagrams, much less between mappings and the chemical systems that form the basis for their own work (p. 169). Yet connections can be and indeed have been made between self-organizing processes and dynamical systems theory. Roux, Rossi, Bachelart, and Vidal ( 1980 ) have written about the BZ reaction in terms of its being a "strange attractor"; Hudson and Mankin (I $98 \mathrm{I}$ ) have

"Prigogine and Stengers contributed a long commentary on Michel Serres's essay "Lucretius: Science and Religion," in Hermes: Literature, Science, Philosophy (Baltimore: Johns Hopkins University Press, 1982). 
discussed the chaotic behavior of the well-stirred version of the reaction; and Simoyi, Wolf, and Swinney (I 982) have compared the behavior of the $\mathrm{BZ}$ reaction to period doubling in one-dimensional maps. However, there is an obvious and important difference between these analyses and Prigogine and Stengers's approach. Prigogine and Stengers emphasize that a large entropy production results in the creation of macroscopic structures where none had existed before. In strange-attractor analyses, the form is considered to be encoded within the information the system produces, but these patterns do not necessarily result in stable, self-replicating structures as such.

I want to emphasize that these are criticisms of Prigogine and Stengers's presentation, not of the importance of self-organizing structures. Since Order out of Chaos was written (the original French version appeared in 1979), significant advances have been made in understanding how self-organization occurs and how the simpler systems can be modeled. Madore and Freedman (1987) have created two-dimensional computer simulations of the $\mathrm{BZ}$ reaction using a cellular automata model that has suggestive implications for some of the larger issues Pirgogine and Stengers raise. It may be helpful to compare and contrast this research with the claims of $\mathrm{Or}$ der out of Chaos, for it illustrates how a more conservative, nonphilosophical approach nevertheless supports the overall thesis about the importance of self-organizing reactions. At the same time, this approach reveals how many links are missing from the causal chain Prigogine and Stengers would forge between the mechanics of self-organizing reactions and a metaphysical privileging of becoming over being.

In the cellular automata approach, the simulation space is divided into cells, and each cell is assigned to one of three states: active, receptive, or quiescent. An active cell can excite an adjacent cell if the adjacent cell is in a receptive state. After a prescribed time, active cells decay into quiescence. After a still longer time, quiescent cells can become receptive again. These sequencing rules mean that active states cannot propagate into areas that are already active, nor can they propagate into quiescent areas.

Suppose that, in the center of the simulation space where all the cells are in a receptive state, a single cell is made active. Excitation 
will spread outward in a circle from this point. Because active cells decay into quiescence, the cells closest to the point of excitation will quickly become quiescent and will be unable to be activated again immediately. This process provides a "pushing" force that makes the circle into a ring and keeps it radiating outward. If the ring is disturbed or fractured along its perimeter, spiral arms appear of the form observed in the $\mathrm{BZ}$ reaction. Virtually all of the spectacular displays observed in the $\mathrm{BZ}$ reaction can be recreated by this simple model (Cohen, Neu, and Rosales, I978; Duffy, Britton, and Murray, 1980). Recent work has expanded the approach to three-dimensional models of the $\mathrm{BZ}$ reaction (Welsh, Gomatom, and Burgess, I 983; Winfree and Strogatz, I 985 ).

This propagating mechanism may explain how complex spatial organizations can spontaneously come into existence. Spiral forms similar to those observed in the $\mathrm{BZ}$ reaction have been found, for example, in nerve axons (Madore and Freedman, I987), in the lens of the firefly's compound eye (Winfree, I980), and in slime mold aggregations (Newall and Ross, i982). This last example is particularly interesting because it is possible to observe the spiral configurations as they form. Slime molds reproduce through spores, each of which is a one-celled organism. As long as there is abundant food in the environment, the mold stays in this form. As soon as the food supply is exhausted, however, the spores swarm together and take on specialized functions, eventually forming a stalk that in turn gives out spores again. A crucial step in the mold's transformation from randomly distributed cells to a complex organized structure is the formation of spiral configurations around the individual spores. Thus it appears that the morphogenic tranformation is achieved through a self-propagating process similar in configuration to the $\mathrm{BZ}$ reaction.

A cellular automata model has also been used to explain largescale spatial organizations, such as the spiral arms of a disk galaxy (Mueller and Arnett, 1976). Of course, many more perturbations are present in galactic systems than in the small petri dishes where the BZ reaction takes place. To account for these perturbations, stochastic elements are introduced into the simulation, giving the spiral arms the "raggedness" characteristic of galaxy formations. The claim of researchers who model systems in this way is not that these 
formations are all the same, only that they employ mechanisms that are similar in that they achieve a "pushing" force through the interplay among active, receptive, and quiescent areas. The forces responsible for creating these spatial gradients in interstellar gases are obviously very different from those responsible for giving the $\mathrm{BZ}$ reaction its characteristic forms. The models thus suggest how morphogenesis can occur, but they do not explain the particular forces at work in a given situation.

As we shall see in chapter 7 , this kind of modeling is typical of dynamical systems methods. The procedure raises questions about what it means to model a system, and ultimately about the nature of scientific explanation. The fact that these models have little or no relation to specific mechanisms makes it easier to justify injecting stochastic elements into them, for the absence of a causal explanation makes the intrusion of chance conceivable at any point. Thus Prigogine and Stengers's valorization of chance is implicit in these modeling procedures, although in dynamical systems methods it is presented as a pragmatic technique rather than as a philosophical revolution.

Given Prigogine's interest in the arrow of time, it is perhaps not surprising that he would turn to cosmology, for the fundamental parameters of the universe were no doubt set at its birth. Edgard Gunzig, Jules Geheniau, and he have recently proposed a model that makes the order-out-of-chaos dynamic intrinsic to the formation of the cosmos (1987). This model imagines that at the very beginning of the universe, fluctuations in the quantum vacuum stabilized themselves in the form of small black holes, which in turn became the matter generators necessary to explain why there is something rather than nothing. "As far as energy is concerned," Prigogine and his coauthors write, "the creation of the universe from a Minkowskian [quantum] vacuum is indeed a 'free lunch" (p. 623). In this model, the scenario Kelvin imagined for the universe is completely reversed. "Heat death" occurs at the beginning rather than the end of time. Thus the transformation of entropy from epilogue to prologue is complete, and chaos is reinstated as our primordial parent.

To see how the model works, it will be useful to explore some of the questions left unanswered by big-bang cosmologies. A big-bang model predicts that residual background radiation would be left 
over from the explosion. This radiation was found (inadvertently) by Arno Penzias and Robert Wilson in 1965 , when they were testing a very sensitive microwave detector for Bell Laboratories and could not get rid of residual noise. No matter in which direction they pointed the detector, the noise remained the same, an indication that it must be coming from outside the atmosphere. Moreover, the radiation remained at the same level, regardless of the time of day or year it was measured. Penzias and Wilson eventually realized that they were detecting the background radiation predicted by big-bang cosmologies. But the extraordinary constancy of the background radiation was a surprise. Repeated observations have confirmed that it does not vary by more than one part in ten thousand. This consistency is striking evidence for the so-called cosmological principlethe premise that the universe is isotropic (that is, does not vary with the axis of observation) and homogeneous.

Why it is surprising that the background radiation should be so uniform? According to the theory of relativity, if light cannot reach a region, nothing else can either, because nothing can travel faster than the speed of light. There would not have been time in the early universe for light from one region to travel to all the other regions, and vice versa. Why then should the background radiation be constant throughout the universe, since the various regions could not have communicated with each other to achieve equilibrium? And why, in contrast to the remarkable homogeneity of the background radiation, should matter be clumped into stars and galaxies?

To answer these questions, Alan Guth and others proposed a "new inflationary cosmology," in which the universe was supposed to have expanded initially at an exponentially greater rate than it does at present. ${ }^{6}$ The increased expansion rate of an inflationary cosmology means that a large part of the universe's present dimensions would have been achieved in a mere fraction of a second-that is, in a much, much shorter time than if the expansion rate remained unchanged. Therefore light would have had time to travel throughout

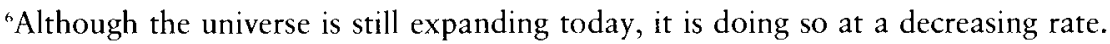
One of the unanswered questions of contemporary cosmology is whether the universe will eventually stop expanding and begin to contract. Prigogine's model predicts that the expansion will continue, causing the universe to generate new matter in the far future.
} 
the universe, and thus we have an explanation for why the universe is isotropic and homogeneous.

Although it answered some of the questions left unresolved by the big bang, the new inflationary cosmology raised new questions of its own. Since the expansion rate is presumed to have changed, some mechanism is needed to explain why it slowed down. The explanations that were advanced relied on special circumstances that looked suspiciously like special pleading. These problems led Stephen Hawking, in A Brief History of Time (I 988), to pronounce "the new inflationary model dead as a scientific theory, although a lot of people do not seem to have heard of its demise and are still writing papers as if it were viable" (p. I 32). Among the people who believe reports of its death to be greatly exaggerated are Gunzig, Geheniau, and Prigogine. Ironically, they use ideas Hawking helped develop to overcome objections to an inflationary cosmology.

Hawking points out that although the general theory of relativity leads to predictions of a big bang, the theory cannot be considered a complete explanation of why a big bang would occur, for two reasons. First, it imagines a situation in which particles would be so tightly squeezed together that interactions between them could not be ignored, making quantum mechanics necessary. Second, it concedes that under the kind of compression the big bang imagines, the known laws of physics would break down. Hawking was looking for a way to combine quantum mechanics with relativistic cosmology when he was led to the realization that black holes could emit radiation. In this case, as he puts it, "black holes ain't so black."

Black holes are relevant to big-bang cosmologies because both represent singularities in spacetime. A black hole is formed when a star larger than a certain mass begins to burn out. Without the fuel to maintain itself, the star experiences massive gravitational collapse, and the matter within it becomes extremely dense. This dense matter in turn creates a gravitational field so intense that it creates a singularity in the curvature of spacetime. To visualize this process, imagine pushing the point of a pencil into an inflated balloon (but not so hard that the balloon breaks). The balloon represents the normal curvature of spacetime. The indentation into which the now-invisible pencil point disappears represents the black hole. The pencil point rests on the bottom of the black hole, the point of the singu- 
larity where the gravitational field is most intense. On the balloon's surface can be seen an indented circular area whose curvature becomes steeper as it disappears inward. For a black hole, the circumference of this curved area is called the event horizon. Once light or anything else goes past the event horizon, the curvature is so steep that it will never be able to escape. Hence the circumference of the event horizon defines what can happen near the black hole and still remain knowable. Once inside the event horizon, events are intrinsically unknowable because light, and hence any other information, cannot escape the gravitational field to return to an observer.

Hawking introduces the idea that black holes might not be black by asking what would happen if we threw something high in entropy-say, a box full of an ideal gas-down a black hole (pp. I02ro5). Would the second law be violated? Clearly, the entropy outside the black hole would decrease. But the second law could be satisfied if we supposed that the entropy inside the black hole increased by a commensurate or greater amount. This supposition implies that it would be convenient to have some way to measure a black hole's entropy-a difficult task, since no direct measurements are possible.

Because Hawking believed that nothing could emerge from a black hole, he had earlier proposed that the event horizon of a black hole could only increase, never decrease. This formulation is reminiscent of the second law's decree that the entropy of a closed system always tends to increase, never decrease. Hawking recounts his feeling of annoyance when Jacob Bernstein, a research student at Princeton, published a paper suggesting that the area of a black hole's event horizon could be taken as a measure of its entropy. In a sense Bernstein's thesis solved Hawking's problem, for it provided a measure of a black hole's entropy. But in another sense it created problems for Hawking's theory. If a black hole has entropy, then it should also have a temperature (since entropy is defined as a function of temperature). And if it has a temperature, then it must emit radiation (otherwise, it could remain at the same temperature forever, in violation of the second law). But if it emits radiation, then it can't be black, can it?

The solution to the dilemma, Hawking explains, was to suppose that "the particles do not come from within the black hole, but from 
the 'empty' space just outside the black hole's event horizon!" (p. I05). He reasoned that if the space around a black hole were really empty, the gravitational and electromagnetic fields would have to be exactly zero. But in this case their values could be known simultaneously and exactly, a result prohibited by the uncertainty principle. ${ }^{7}$ Hence there have to be fluctuations within these fields, which can be thought of as the creation of virtual particle pairs. Unlike real particles, virtual particles cannot exist independent of each other. When they take the form of matter, they come into existence as a particle and antiparticle and immediately annihilate each other. If these particle pairs came into existence at the boundary of a black hole, however, one of them could fall into the hole. Then the other could go free and become a real particle.

In order for a black hole to be an effective particle catcher, it would have to be very small, for the particle pair is separated by only a very short distance. Hawking calculated that a small black hole with a mass of a thousand million tons (equivalent to a large mountain) would have an event horizon diameter of a millionth of a millionth of an inch, about the size of an atom's nucleus (p. I08). Black holes of this diameter are not formed by collapsing stars. They could, however, have been formed at the universe's creation, when pressures and temperatures were sufficiently intense to bring about the required compression. Small black holes differ from large ones in the higher temperature at which they emit radiation. Because radiation energy cannot come into existence from nothing, it must be balanced by a loss of mass within the black hole. ${ }^{8}$ As the black hole shrinks, it emits radiation at a still higher energy, and so shrinks even faster. Thus small black holes operate according to a dynamic in which an initially small perturbation drives the system further from equilibrium, which increases the perturbation, which drives it yet further. This accelerating feedback loop means that small black holes could eventually "evaporate." Although no one knows exactly

The strength and location of the field are conjugate variables, and hence are analogous to the position and momentum of a particle, which are more usually mentioned in relation to the uncertainty principle. In its more general form, the uncertainty principle applies to any appropriate pair of conjugate variables.

'By Einstein's equation $E=m c^{2}$, energy is proportional to mass. The radiation streaming out from a black hole is balanced by matter conversion within it. 
what happens when a singularity in spacetime pops back out (as the balloon pops back into place when the pencil is removed), the most likely scenario calls for a final burst of radiation as the black hole disappears completely. If primordial black holes do exist," Hawking points out, they would "hardly deserve the epithet black: they really are white hot and are emitting energy at the rate of about ten thousand megawatts" (p. Io8).

With this preamble, we are now ready to understand how Prigogine and his co-authors appropriated Hawking's ideas while changing their drift. They propose a cosmological model in which primordial black holes are created not through the big bang but through the fluctuations in the quantum vacuum which Hawking argued had to be present to keep the uncertainty principle from being violated. Because these black holes have masses even smaller than the ones Hawking imagined (by a factor of ten), Gunzig, Geheniau, and Prigogine call them a "dilute black hole gas" (1987: 623). These extra-small black holes would operate through the same unstable dynamic that Hawking proposed, whereby the black hole generates more radiation and brings more particles into being the smaller it becomes. Hence the clumpiness of matter, which is formed as particles coalesce around the black hole gas. A neat feature of the model is the explanation it provides for why the universe's initial inflationary rate changed. The phase transformation falls out of the equations as a result of the evaporation of the black hole gas! Moreover, the time required for the evaporation matches the time necessary for the universe to expand at an increased rate if it is to be homogeneous. Thus the model explains two features of the present universe which big-bang cosmologies cannot-its isotropy and the uneven distribution of matter within it.

An important difference between this model and Hawking's is that large entropy production is built into the universe from its beginning. As the black holes evaporate, their entropy becomes, in ef-

\footnotetext{
"To date, the existence of such primordial black holes has not been confirmed, despite attempts to locate them. Search strategies have focused on detecting them through the $\mathrm{X}$ rays and gamma rays they would emit. Gamma rays do not penetrate the earth's atmosphere; hence a detector must be positioned in space. Moreover, there are other sources of gamma rays around, so it would be necessary to establish that the detected gamma rays came from a single source. These difficulties are perhaps sufficient to explain why none has been observed.
} 
fect, the entropy of the universe. Another distinctive feature of the model is the prediction that as the universe continues to expand, it will eventually reach the point where it is sufficiently attenuated so that the quantum vacuum will again bring more black hole gas into existence, which will in turn generate more matter. Thus, although the universe will continue to expand, it will never reach a point of ultimate dissipation because it possesses the capacity to renew itself. Clearly this model would appeal to Prigogine, for it inscribes the order-out-of-chaos scenario into the creation of the universe. What greater validation of the paradigm than to have it explain why there is something rather than nothing? Not coincidentally, it also weaves the second law into the very fabric of the universe, making it coeval with the universe's formation rather than an artifact added later. Thus Prigogine is able to find in cosmology the answer that eluded him in Order out of Chaos.

Such deeply felt considerations as I presume lie behind Prigogine's foray into cosmology do not, obviously, prove the model's validity. But neither do they disprove it. One could argue that significant scholarship in any field is compounded with personal emotion, for why would someone devote to it the countless hours that stretch across months, years, decades, unless it mattered on more than a strictly intellectual level? The fact that Prigogine's work evidently emerges from a vision of the way the universe could or should be, rather than the vision emerging from the work, is reflected in the apparent imbalance between the practical consequences of his theories and the dramatic claims he makes for them. Peter V. Coveney, in a recent review of Prigogine's work which was largely sympathetic (I 988 ), acknowledges the problem.

A criticism that has not infrequently been aimed at the work of the Brussels School ... is that, even if it is granted from a philosophical standpoint that irreversibility should be included in the fundamental level of description, there do not appear to be any new and experimentally verifiable consequences to have emerged from the approach. If this were the case, the proclaimed "revolution" in scientific throught arising from the advent of an adequate role for irreversibility and time... would hardly be valid.

Coveney, who lists the Université Libre de Bruxelles as one of his affiliations, concludes that he believes the criticism has some merit, 


\section{Ir2 Something out of Nothing}

but hopes "it will be rectified by consideration of problems of concrete physical interest" (p. 4I4).

The question of what weight should be given to Prigogine's vision, as distinct from "problems of concrete physical interest," goes to the heart of why his reputation within the scientific community is so uneven. For scientists concerned with the practical problems that make up 90 percent-or 99 percent-of scientific research, Prigogine's work appears to be so much useless metaphysical speculation. From this perspective, his reputation appears to be grossly inflated. But there have been times when vision was central, as in Einstein's quarrel with quantum mechanics, which he pursued in the face of strong evidence against his position because he felt sure that God did not play dice with the universe. Another case in point is Bacon's Novum Organum. In spite of being wrong in nearly all of its specific predictions, this text has endured because it had a vision of what science could be. The examples illustrate that at pivotal moments, vision can become the essence of what is at stake, and practical problems can be secondary or even irrelevant.

Disputes about vision do not tend to occur when science is in the "normal" phase that Thomas Kuhn ( 1970) has identified with the presence of a satisfactory intact paradigm. When such disputes do break out, they often indicate that the prevailing paradigm is under pressure. If the pressure mounts to a crisis, presuppositions can come into view which were invisible during the normal phase. Order out of Chaos is an attempt to articulate and refute some of the presuppositions of classical physics. If the mere existence of this book suggests that a paradigm shift is in progress, its mixed reception indicates how far such a shift has yet to go. From talking with scientists who work with complex dynamical systems, I know that many of them regard Prigogine's claims as overstated. Their main interest is in what the new techniques will allow them to do with problems of interest to them. Their involvement with practical problems may partially conceal a paradigm shift from them, for it is often the case that new techniques evolve first, and then attitudes change later as their deeper implications are recognized. I therefore do not rule out the possibility that Prigogine and Stengers's argument may turn out to be correct in its general thrust, even if it is wrong or misleading in its specifics. 
The concatenation between a proposed solution to an intellectual problem and a strongly felt personal significance is also evident in Hawking's book. He makes clear that for him, the prospect that the known laws of physics break down at the big-bang singularity is deeply distressing, for he believes that the universe should be knowable by human beings. When he discusses the possibility that the universe is just a "lucky chance," he labels it "the counsel of despair, a negation of all our hopes of understanding the underlying order of the universe" (I988: 133). Later, to introduce the mathematical transformation that he asserts can remove the big-bang singularity, he points out that the "idea that space and time may form a closed surface without boundary also has profound implications for the role of God in the affairs of the universe. . . So long as the universe had a beginning, we could suppose it had a creator. But if the universe is really completely self-contained, having no boundary or edge, it would have neither beginning nor end: it would simply be. What place, then, for a creator?" (pp. I40-I4I). Such remarks lead one to believe that Hawking's dissatisfaction with big-bang cosmologies is commingled with questions about God, and that the range of possible solutions is constrained by the kind of God he can accept-namely, a God who agrees in advance to make the universe understandable at every point by human beings. ${ }^{10}$

In this respect Hawking is no different from Prigogine or Einstein. Each is motivated by a vision that underlies and guides his scientific research. How to judge between competing visions? It is often said that the art of scientific inquiry lies in asking questions that can be answered. The general theory of relativity, for example, enabled researchers to ask whether light would be bent by the gravitational field of the sun. When the answer turned out to be yes, it was an important confirmation of the theory's validity.

What this formulation obscures, however, is that the visions that guide theory and research involve issues that are larger than answerable questions can address. Light may be bent by gravitation, but this finding does not answer the question whether irreversibility is intrinsic to reality. Self-organizing processes may take place, but this finding does not answer the question whether chance is to be cele-

\footnotetext{
${ }^{10} \mathrm{I}$ am indebted to Clair James of the University of Iowa for suggesting this idea.
} 


\section{I4 Something out of Nothing}

brated. A mathematical transformation that removes the big-bang singularity may exist, but this finding does not answer the question whether the universe is ultimately knowable by human beings. Experimental results can help to shape, refine, and substantiate a vision, but they are not usually sufficient to determine it.

Where does the vision come from? No doubt the circumstances of an individual life play an important role. But visions take hold and spread because they speak to something in the cultural moment. They signify more than the research can demonstrate; and it is this excess signification that produces and is produced by the cultural matrix. Prigogine's vision is of a universe rich in productive disorder, from which self-organizing structures spontaneously arise and stabilize themselves. His vision imagines the cosmos providing, as Alan Guth says, "the ultimate free lunch"-a gourmandizing delight where the pot is never empty, and where the flow of time leads to increasing complexity rather than decay. ${ }^{11}$ The vision sees nothingness and somethingness joined in a complex dance, in which vacuums are never truly empty and gaps are never merely ruptures.

To some extent Henry Adams felt the power of this vision, although when he wrote it was only a glimmer of a possibility, and his circumstances led him to cast it in a darker mold than does Prigogine. Stanislaw Lem also shares parts of the vision. More contemporary than Adams and more literary than Prigogine, Lem connects the image of the creative void with poststructuralist theory. If these convergences suggest that the vision is more widely shared than Prigogine's critics acknowledge, they also demonstrate that the values associated with it are more diverse and polysemous than Prigogine recognizes.

"Guth's remark is quoted in Hawking, x $988: 129$. 